Short-Term Effects

of Intratracheal Installations

of Yttrium Barium Copper Oxide

I. E. London

L. R. Newkirk

B. E. Lehnert 


\title{
SHORT-TERM EFFECTS OF INTRATRACHEAL INSTALLATIONS OF YTTRIUM BARIUM COPPER OXIDE
}

by

\author{
J. E. Lundon, L. R. Newkirk, and B. E. Lehnert
}

\begin{abstract}
Inhalation exposures to the new high-temperature ( $T \partial$ superconductor (SC) materials can occur during manufacturing and fabrication processes. In this exploratory study, we examined the pulmonary response to the deposition of an yttrium barium copper oxide SC powder. Groups of Sprague-Dawley rats were intratracheally instilled with either $10 \mathrm{mg}$ or $20 \mathrm{mg}$ of SC in phosphate-buffered saline (PBS) or with PBS only. The animals were sacrificed 60 days later for histopathologic assessments of their lungs. Lung lesions in the 10-mg SC group were found mainly in alveolar ducts and proximal alveoli. The lesions consisted of variably sized foci of interstitial thickening involving accumulations of macrophages. These interstitial aggregates were often times centered around one or more extracellular crystals, which, presumably, were retained SC product. Trichrome stains also demonstrated the presence of fibrosis in ihe walls of alveoli suriounding the granulonıas. Similar interstitial-macrophage accumulations and fibrosis were observed in rats instilled with $20 \mathrm{mg}$ of SC. However, alveolus like structures lined by ciliated cuboidal epithelium . 1ear interstitial granulomas were additionally found in the lungs of some of the anirials in this latter group. Thase results suggest SC material of the yttrium barium copper oxide type may represent a potential exposure hazard to the lung.
\end{abstract}




\section{INTRODUCTION}

Ongoing progress in the development of new materials with high-temperature ( $T \partial$ superconductor properties is anticipated to result in the commercial application of these materials in the near future. Human exposures to these advanced materials, accordingly, will potentially occur during their production, fabrication, and application. Thus, a data base of the potential health risks introduced by emerging SC technology is required to identify workplace and application constraints.

As a part of the Cellular and Molecular Biology (LS-4) Group's applied toxicology program, yttrium barium copper oxide was examined to define its toxic properties with the following tests: (1) acute oral toxicity, (2) primary skin irritation, (3) skin sensitization, (4) eye (conjunctival) irritation, and (5) the pulmonary response to the material in the rat. ${ }^{1}$ This report focuses on our findings regarding the pulmonary toxicity of the test material.

\section{METHODS}

\section{Superconductor Material}

The test powder used in the study was yttrium barium copper oxide $\left(\mathrm{YBr}_{2} \mathrm{Cu}_{3} \mathrm{O}_{7}\right.$. x) obtained from Grace \& Co., Baltimore, Maryland (Lot 14666-14-22A). The relative abundances of ytrrium, barium, and cooper by weight were $15.6 \%, 49.7 \%$, and $34.7 \%$, respectively. The powder was black in appearance; and, according to the manufacturer, it had a density of 6.256 and a surface area of $0.390 \mathrm{~m}^{2} / \mathrm{g}$. The median size of the particles composing the powder was $6.6 \mu \mathrm{m}$ within a size range of 0.4 to $100 \mu \mathrm{m}$. According to the manufacturer, the material is "soluble in acid." 
Animals and Intratracheal Instillations

Adult, male Sprague-Dawley rats were randomly assigned to three groups. One group were intratracheally instilled with $10 \mathrm{mg}$ of superconductor (SC) material suspended in $0.4 \mathrm{ml}$ of phosphate-buffered saline (PBS). Another group of rats were instilled with $20 \mathrm{mg}$ of the material in $0.4 \mathrm{ml}$ of PBS. The third group of animals were instilled with $0.4 \mathrm{ml}$ of PBS only. All instillations were performed on Ethrane $e^{{ }^{*}}$. anesthetized rats. Two rats that were instilled with the $20-\mathrm{mg}$ lung burden of particulate SC died within hours thereafter.

\section{Animal Sacrifices and Lung-Tissue Processing}

Rats from all three groups were sacrificed by means of intraperitoneal (IP) injections of pentobarbital sodium $(50 \mathrm{mg}$ ) on day 60 following the instillations. The lungs were excised, filled with 10\% formalin in PBS, and the left lung lobes were embedded in paraffin. Regional tracheobronchial lymph nodes were also fixed and processed for histopathologic analyses. Tissue sections $(4 \mu \mathrm{m})$ were stained with hematoxylin and eosin (H\&E) or with Masson's trichrome stain (MTS) as a means to detect the abnormal accumulation of lung collagen. The lungs of the rats that died shortly after they were instilled with SC were also prepared for histologic examination.

\section{RESULTS}

\section{Lung Histepathelogy}

Occasional single foci of alveolar histiocytosis were observed in the lungs of some (two out of five) of the PBS-instilled animals. Otherwise, the control lungs were unremarkable in appearance.

\footnotetext{
"Ethrane is a registered trademark of Anaquest, Arlington, Texas.
} 
As previously indicated, two animals died shortly after instillation of the 20mgmass burden of SC. The large airways in the lungs of these animals were filled with the material, suggesting that these rats died as a result of airway occlusion by the material. At the sime of death, the instilled material also lined many alveolar ducts and was contained in surrounding alveoli. Capillaries in these regions were congested, and prominent perivascular edema was also observed around small arterioles adjacent to the terminal bronchioles.

Lesions produced by the SC material ( 10 or $20 \mathrm{mg}$ ), as of day 60 after it was instilled, generally centered in the periterminal bronchiole-alveolar duct-alveolar region. The response included variably sized foci of interstitial thickening caused mainly by interstitial accumulations of macrophages. Small numbers of polymorphonuclear leukocytes were also observed in the foci with the macrophages in lungs that received the $20 \mathrm{mg}$ burden of SC. Another major component of the lesion was the intra-alveolar accumulation of alveolar-macrophage aggregates and aggregates of interstitial macrophages. Typically, the granulomatous response was more pronounced in the lungs of rats instilled with the 20-mg dose than it was in lungs instilled with the 10-mg burden of SC material (Table I).

The interstitial-macrophage aggregates were often centered around one or more apparently extracellular crystals, which were also observed in the alveoli and occasionally in the aggregates of alveolar macrophages. The crystals varied in size, shape, and staining characteristics. Most appeared as small plaques of irregular shape with either eosinophilic or basophilic staining characteristics. At some sites, a dark brown material was found admixed with the more typical plaquelike crystals. The crystals, which probably were of SC origin, were not birefringent under polarized light. 


\section{Table 1}

Comparative Severities of Granulomatous Responses in the Lungs and Lymph Nodes Relative to Instilled Mass of SC Materialsa

Treatment

Group

Control Rats

1

2

3

4

5

10-mg SC Rats

1
2
3
4
5
6

20-mg SC Rats

1

2

3
6
Granulomatous

Pneumonia ${ }^{b}$
Granulomatous

Lymphadenitis ${ }^{c}$
0

$0^{d}$

0

0

0

$\begin{array}{ll}0 & 0 \\ 0 & 0\end{array}$

$+$

$+$

$++$

$+$

$+$

$+$
$+$

$++$

$+$

$++$

$++$

$++$

a Legend: + mild, ++ moderate, +++ severe.

bpneumonia, granulomatous, multifocal.

CLymphadenitis, granulomatous, multifocal.

dHistiocytosis, focal, moderate. 
Examination of tissue sections stained with $\mathrm{H} \& \mathrm{E}$ indicated that abnormal deposition of collagen was present within the granulomas. MTS confirmed the presence of mild to moderate fibrosis in areas of macrophage aggregation. In addition, the MTS revealed the occurrence of fibrosis within the walls of alveoli surrounding granulomas, even when the walls contained minimal numbers of macrophages.

Unusual foci of alveoluslike structures lined with ciliated cuboidal epithelium were also observed to be associated at sites adjacent to interstitial granulomas in two of the three animals that were instilled with $20 \mathrm{mg}$ of SC material.

\section{Lymph-Node Histepathology}

Generally, the intrapulmonary deposition of the SC material (10- or $20-\mathrm{mg}$ burdens) resulted in enlargements in the tracheobronchial lymph nodes. In many nodes, the medullae appeared smaller than in the control nodes, whereas the paracortices were larger than the control condition. Multiple prominent foci of aggregated macrophages, which were of variable size and shape, were observed throughout the nodes, although such aggregates were relatively less frequent in the superficial cortex than in the paracortex and medulla.

Macrophages composing the granulomatous foci in the nodes appeared to contain crystals of foreign material that were generally smaller and more eosinophilic thain the crystals seen in the lung sections. MTS did not reveal an unusual amount of connective tissue in the nodes of the animals instilled with the SC material.

\section{SUMMARY AND DISCUSSION}

* The pulmonary response to the deposition of SC material observed in this study is best described as granulomatous pneumonia. Unlike granulomatous pneumonias caused by infectious agents, however, the granulomatous process induced by SC 
powder does not appear to involve interstitial accumulations of lymphoid cells. Instead, virtually the only inflammatory cell present in the interstitial lesion was the macrophage.

* The absence of clearly evident giant-cell formation in the SC-induced granulomatous response is unusual in cases of granulomatous pneumonia. Type ॥ pneumocyte hyperplasia, which is a typical feature of chronic pneumonia, also was not observed in this study.

* MTS indicated the occurrence of fibrosis in the lung in response to SC deposition.

* The appearance of ciliated epithelium in alveoluslike structures following the intrapulmonary deposition of SC material may represent a form of metaplasia or regeneration in response to the powder. Similar histologic abnormalities have been associated with the deposition of high burdens of other types of particles in the lung. Bronchiolepithelialization of the alveoli is also known to oscur before the development of lung tumors in response to some carcinogenic agents.

* The response in the regional lymph nodes of animals instilled with SC material is consistent with granulomatous lymphadenitis caused by the presence of a foreign substance. Histologic evidence obtained in this study indicated that the SC material, or at least a form of the material, can translocate from the lungs to the lymph nodes. Whether the material passes to the nodes in free form or by a macrophage-mediated process, or both, remains to be determined. Regardless, unlike in the lung, a fibrogenic response was not observed in the nodes. 


\section{ACKNOWLEDGMENTS}

The authors express their sincere appreciation to Donna Kusewitt for the histopathology evaluation.

1. J: E. London, "Preliminary Toxicological Study of Yttrium Barium Copper Oxide," Los Alamos National Laboratory report LA-11437-MS (Dec. 1988). 

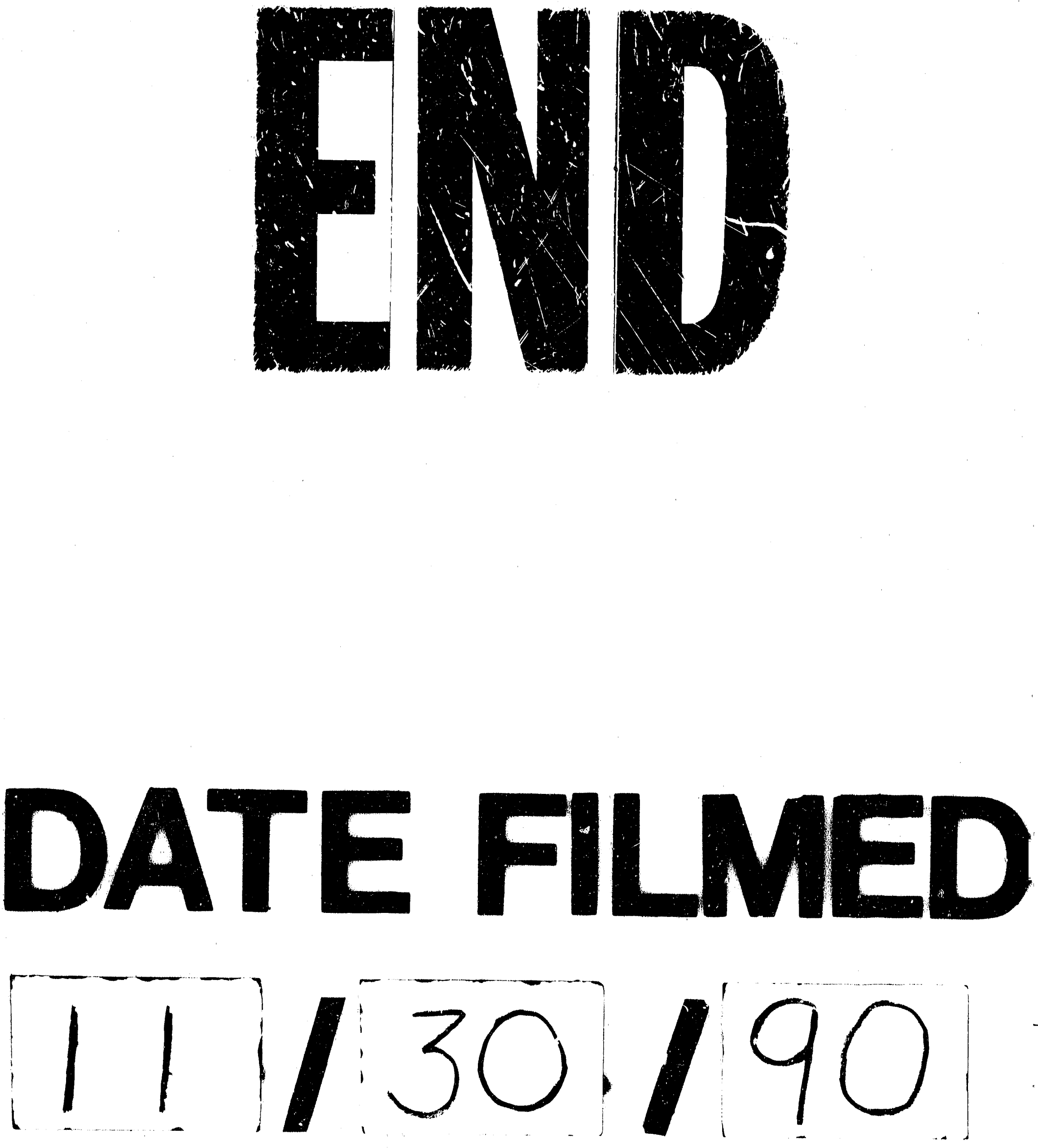
